\title{
Mecoptera (Insecta) do estado do Maranhão: chave para identificação das espécies e descrição da fêmea de Bittacus latreillei (Collucci \& Amorim)
}

\author{
Renato José Pires Machado ${ }^{1,2}$, Francisco Limeira-de-Oliveira ${ }^{3} \&$ José Albertino Rafael $^{1,4}$
}

${ }^{1}$ Coordenação de Pesquisas em Entomologia, Instituto Nacional de Pesquisas da Amazônia - INPA, Av. André Araújo, 2936, Aleixo, 69060-001 Manaus-AM, Brasil.

${ }^{2}$ Bolsista do Programa de Capacitação Institucional - PCI, MCT/CNPq/INPA. rjpmachado@gmail.com

${ }^{3}$ Laboratório de Estudos dos Invertebrados, Universidade Estadual do Maranhão (UEMA), Praça Duque de Caxias, s/nº, Morro do Alecrim, 65604-380 Caxias-MA, Brasil. franciscolimeira@cesc.uema.br

${ }^{4}$ Bolsista do CNPq; jarafael@inpa.gov.br

\begin{abstract}
Mecoptera (Insecta) from Maranhão state: identification key for species and description of the female of Bittacus latreillei (Collucci \& Amorim). Recent field collections in Maranhão state, Brazil, indicate that the mecopterans seem to be abundant in this state. 55 specimens of four species were studied: Bittacus diversinervis Souza Lopes \& Mangabeira, 1942, B. femoralis Klug, 1836, B. latreillei (Collucci \& Amorim, 2000) and Issikiella araguaiensis Penny \& Arias, 1983. Most of the specimens were collected between October and February, the rainy season in Maranhão. An identification key for these species and the description of the female of $B$. latreillei are presented.
\end{abstract}

KEYWORDS. Bittacidae; Brazil; hangingflies; Northeast region; seasonality.

RESUMO. Mecoptera (Insecta) do estado do Maranhão: chave para identificação das espécies e descrição da fềmea de Bittacus latreillei (Collucci \& Amorim). Coletas recentes no estado do Maranhão indicaram que os mecópteros parecem ser abundantes neste estado. Foram estudadas 55 espécimes de quatro espécies: Bittacus diversinervis Souza Lopes \& Mangabeira, 1942, B. femoralis Klug, 1836, B. latreillei (Collucci \& Amorim, 2000) e Issikiella araguaiensis Penny \& Arias, 1983. A maioria dos indivíduos foi coletada entre os meses de outubro e fevereiro, período chuvoso no Maranhão. Uma chave de identificação para essas espécies e a descrição da fêmea de $B$. latreillei são apresentadas.

PALAVRAS-CHAVE. Bittacidae; Brasil; região Nordeste; sazonalidade.

Mecoptera é uma pequena ordem de insetos holometábolos que se caracterizam por grandes asas membranosas (de mesmo tamanho e venação) e clípeo e labro geralmente prolongados para baixo formando um rostro (Setty 1940). São relativamente raros e geralmente conhecidos de poucos exemplares (Byers 1965). Atualmente são conhecidas cerca de 600 espécies, distribuídas pelo mundo (Grimaldi \& Engel 2005).

A ordem está dividida em nove famílias (Grimaldi \& Engel 2005), mas somente Bittacidae ocorre no Brasil (Machado et al. 2009). Os bitacídeos são caracterizados pelas pernas raptoriais longas, tarsos com garra única e aparência de Tipulidae (Diptera) (Setty 1940). O pouco que se conhece sobre os seus hábitos tem como fonte os trabalhos de Carpenter (1931), Setty (1940) e Byers \& Thornhill (1983) que focam principalmente as espécies norte-americanas. Os bittacídeos adultos são encontrados em arbustos e plantas herbáceas, onde ficam pendurados pelo primeiro par de pernas esperando insetos pequenos que são capturados, principalmente com as pernas posteriores. As larvas são eruciformes, vivem no solo e são saprófagas; alimentam-se de restos de plantas, minhocas, e de pequenos insetos.

Bittacidae é a segunda maior família da ordem e juntamente com Panorpidae, a mais diversa, possuem cerca de $95 \%$ das espécies descritas (Grimaldi \& Engel 2005). Na região Neotropical é onde está a maior diversidade, sete dos 18 gêneros conhecidos (Machado et al. 2009; Tan \& Hua 2009a, b). Para o Brasil são registradas 23 espécies divididas em quatro gêneros: Bittacus Latreille, 1805 (16 espécies), Issikiella Byers, 1972 (três espécies), Nannobittacus EsbenPetersen, 1927 (duas espécies) e Pazius Navás, 1913 (duas espécies) (Machado et al. 2009).

Entre os trabalhos já publicados sobre os mecópteros brasileiros, nota-se claramente que a região Nordeste é a menos estudada. Os primeiros trabalhos se concentraram nas regiões Sudeste, Sul e Centro-Oeste (Souza Lopes \& Mangabeira 1942; Morgante 1967; Byers 1972). Os subseqüentes voltaram-se, principalmente, para a fauna amazônica (Penny, 1977; Penny \& Arias, 1982). Bittacus diversinervis Souza Lopes \& Mangabeira, 1942, foi a primeira espécie registrada para o Nordeste, coletada em Arcoverde, Pernambuco (Penny \& Arias 1982). Collucci \& Amorim (2001), registraram a segunda espécie para o Nordeste e a primeira para o estado do Maranhão, B. femoralis Klug, 1836, duas fêmeas provenientes de São Luís. Machado et al. (2009) publicaram recentemente uma lista com a distribuição dos mecópteros neotropicais.

A elaboração deste trabalho foi motivada, principalmente, pela carência de estudos relacionados à região Nordeste do 
Brasil e por um surpreendente número de espécimes coletados recentemente na cidade de Caxias e suas proximidades, na região leste do Maranhão, bem como no Parque Estadual do Mirador, região centro-sul do estado.

\section{MATERIAL E MÉTODOS}

Os espécimes foram coletados nas regiões leste e centrosul do estado do Maranhão, onde predomina vegetação de cerrado lato sensu, cuja principal característica estrutural é arbórea, xeromórfica de engalhamento profuso. Este é frequentemente entremeado por matas de galeria, cuja composição florística varia com o local de ocorrência. A temperatura média anual para as regiões leste e centro-sul do Maranhão variam entre $26-27^{\circ} \mathrm{C}$ e $25-26^{\circ} \mathrm{C}$, respectivamente (GEPLAN/UEMA 2002).

Alguns exemplares foram coletados com armadilha luminosa, mas a maior parte dos exemplares estudados foi coletada incidentalmente, sem fazer parte de uma coleta padronizada, principalmente em focos luminosos de edificações urbanas ou rurais.

Aidentificação foi feita por comparações com as descrições originais, espécimes previamente identificados e série tipo. O material está depositado na Coleção de Invertebrados do Instituto Nacional de Pesquisas da Amazônia (INPA), Manaus, Amazonas e na Coleção Zoológica do Maranhão (CZMA), Universidade Estadual do Maranhão, Centro de Estudos Superiores de Caxias, Caxias, Maranhão. Exemplares depositados na coleção da Universidade Federal do Paraná (DZUP) e INPA foram utilizados para facilitar a identificação das espécies.

As fotos que ilustram a chave de identificação foram obtidas com câmera fotográfica digital acoplada a um microscópio estereoscópico. Os desenhos foram realizados em câmara clara acoplada a um microscópio óptico. Alguns espécimes tiveram a terminália dissecada e maceradas em ácido lático $80 \%$ a quente para a realização de ilustrações. Após foram preservadas em microtúbulos com glicerina, alfinetados no indivíduo correspondente.

\section{RESULTADOS E DISCUSSÃO}

Foram analisados 55 espécimes pertencentes a quatro espécies, B. diversinervis, B. femoralis, B. latreillei (Collucci \& Amorim, 2000) e Issikiella araguaiensis Penny \& Arias, 1983 que podem ser identificadas pela chave abaixo.

Chave de identificação das espécies de Mecoptera do Maranhão:

1. Veia $1 \mathrm{~A}$ curta, terminando antes do nível da origem de $\mathrm{M}$; $\mathrm{M}_{1+2}$ mais arqueada que $\mathrm{M}_{3+4}$; veia $\mathrm{Cu}_{2}$ terminando antes ou no nível da primeira bifurcação de M (Fig. 1) ........... Issikiella araguaiensis

Veia 1A longa, passando a origem de $\mathrm{M} ; \mathrm{M}_{1+2}$ e $\mathrm{M}_{3+4}$ igualmente arqueadas; veia $\mathrm{Cu}_{2}$ passando a primeira bifurcação de M (Fig. 2) . (Bittacus)..2

2. Ápice do fêmur e da tíbia e base da tíbia, pretos
Ápice do fêmur e da tíbia e base da tíbia, marrons ....... 3

3. Veia $\mathrm{Rs}_{1}$ bifurcada na asa anterior (Fig. 2). Terminalia masculina: margem posterior dos epândrios em vista dorsal, sem uma projeção lateral (Fig. 5)

B. diversinervis

Veia Rs 1 não bifurcada na asa anterior (Fig. 3). Terminalia masculina: margem posterior dos epândrios em vista dorsal, com uma projeção lateral (Fig. 6) . B. femoralis

\section{Bittacus diversinervis Souza Lopes \& Mangabeira, 1942}

B. diversinervis foi a primeira espécie registrada para o Nordeste brasileiro, em Pernambuco (Penny \& Arias 1982) e sua ocorrência no Maranhão era esperada pois possui ampla distribuição no Brasil, com registros de Roraima a São Paulo. Foram examinados cinco exemplares $(3 \hat{\jmath}$ e 2 ? $)$, todos de Caxias.

\section{Bittacus femoralis Klug, 1836}

B. femoralis era a única espécie registrada para o Maranhão, em São Luís (Collucci \& Amorim 2001). Seu registro ao sul do estado era esperado face à ocorrência também em Goiás, onde predomina o mesmo tipo de vegetação (cerrado). Essa espécie também já foi registrada em Rondônia (Penny \& Arias 1982), além de dois exemplares coletados em Jacaré no Mato Grosso (DZUP) que ampliam sua distribuição geográfica. B. femoralis foi a espécie mais comum entre os espécimes estudados, com 39 exemplares $(18 \hat{\jmath}, 19 ㅇ$ e 2 indeterminados, devido ao abdômen estar quebrado), provenientes dos municípios de Caxias (33), Mirador (5) e São Luís (1).

\section{Bittacus latreillei (Collucci \& Amorim, 2000)}

B. latreillei era conhecido somente pelo holótipo macho proveniente de Mossamedes, Goiás (Colluci \& Amorim

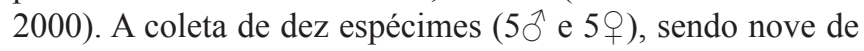
Caxias e um de Presidente Dutra e mais um macho coletado em Óbidos (Pará) depositado na coleção do INPA, amplia significativamente sua distribuição geográfica. O material disponível aumenta o número de espécimes dessa espécie e a captura de fêmeas permite a sua descrição pela primeira vez, a qual é apresentada a seguir.

Descrição da fêmea

Cabeça: fronte, vértice e antenas marrons. Clípeo e labro marrom-avermelhados. Gena, palpos e triangulo ocelar, pretos. Ocelo mediano menor que os dois laterais.

Tórax: marrom com manchas claras centrais na região dorsal. Antepronoto e pós-pronoto com duas ou três cerdas pretas em cada lado. Escutelos com duas cerdas pretas posteriores. Pleura marrom.

Pernas: marrons exceto tarsos, ápice do fêmur e da tíbia e base da tíbia, pretos. A região mediana do fêmur anterior pode ser marrom-avermelhada. Coxas posteriores podem apresentar cerdas pretas. 

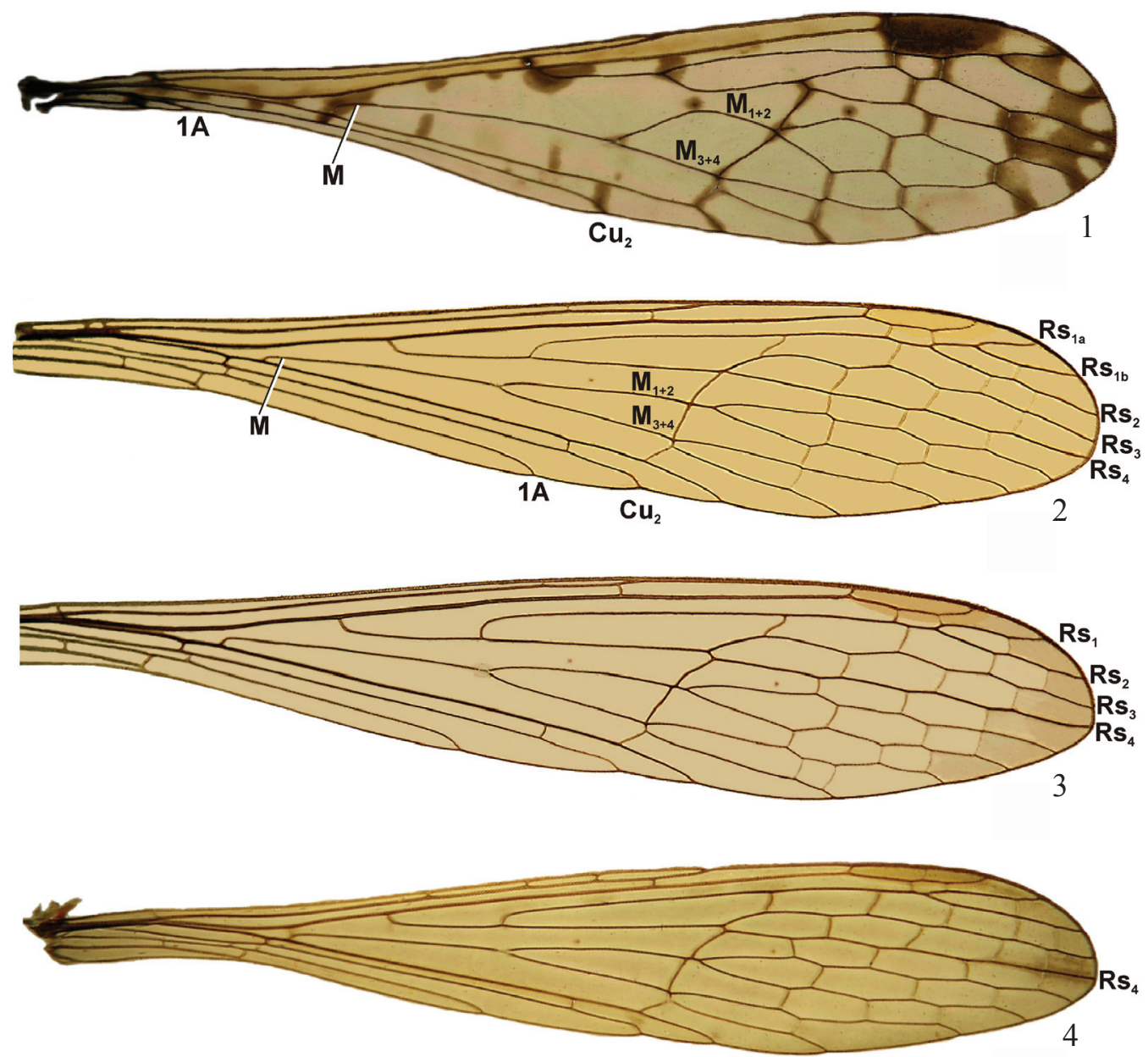

Figs. 1-4. 1, Issikiella araguaiensis, asa anterior; 2, Bittacus diversinervis, asa anterior; 3, B. femoralis, asa anterior; 4, B. latreillei, asa anterior.

Asa (Fig. 4): com membrana amarelada exceto o pterostigma e o ápice de $\mathrm{Rs}_{4}$ na asa anterior, sombreados de marrom. Thyridium evidente. Veias marrons. Veias transversais costais variam de duas a cinco, além da humeral. Duas veias transversais pterostigmais. Pterostigma alongado.

Abdome: marrom, mas o ápice dos tergitos pode ser preto. Terminália (Fig. 7): escleritos e cercos geralmente mais escuros que o restante do abdome. Placa subanal maior que o tergito 11. Cerco terminando aproximadamente no mesmo nível da placa subanal. Gonocoxoesternitos com cerdas pretas grandes na região posterior e não fusionados ventralmente (Fig. 8). Espermateca (Fig. 9) com cápsula volumosa que se estreita na metade basal onde se continua por um tubo uniforme, com aproximadamente metade do comprimento da cápsula e diâmetro maior. Este, por sua vez, continua por um filamento muito estreito, longo e enovelado.

Comprimento do corpo: 17,4 mm-21,6 mm.

Comprimento da asa anterior: $17,1 \mathrm{~mm}-20,4 \mathrm{~mm}$.

Variações: quando comparadas com os machos as fêmeas aqui descritas não apresentam variação. No entanto, os espécimes do Maranhão têm algumas variações em relação à descrição do holótipo como: manchas mais claras na região dorsal do tórax sendo que no holótipo é uniformemente marrom; ápice e base da tíbia pretos, marrons no holótipo; número de veias costais transversais varia de dois a cinco, holótipo com três.

\section{Issikiella araguaiensis Penny \& Arias, 1983}

I. araguaiensis era conhecido somente pela série tipo, toda proveniente de Conceição do Araguaia, Pará (Penny \& Arias 1983). A coleta de uma fêmea no município de Caxias (MA) amplia sua distribuição geográfica. Este é o primeiro registro deste gênero para região Nordeste, antes restrito às regiões Norte e Sul.

\section{Sazonalidade}

Apesar dos exemplares aqui estudados serem coletados aleatoriamente, nota-se claramente que estes são mais comuns no período chuvoso (outubro - fevereiro). Essa sazonalidade dos adultos provavelmente está sendo influenciada pela estação mais úmida. Fora da estação chuvosa, somente dois exemplares de $B$. femoralis foram coletados, em junho.

Essa sazonalidade é semelhante à de $B$. pintoi Souza Lopes \& Mangabeira, 1942, estudada por Dias \& Kumagai (no prelo) em Minas Gerais. As autoras realizaram coletas semanais com armadilhas de interceptação de vôo, tipo Malaise, durante três anos (1998-2000 e 2002-2003) e os 


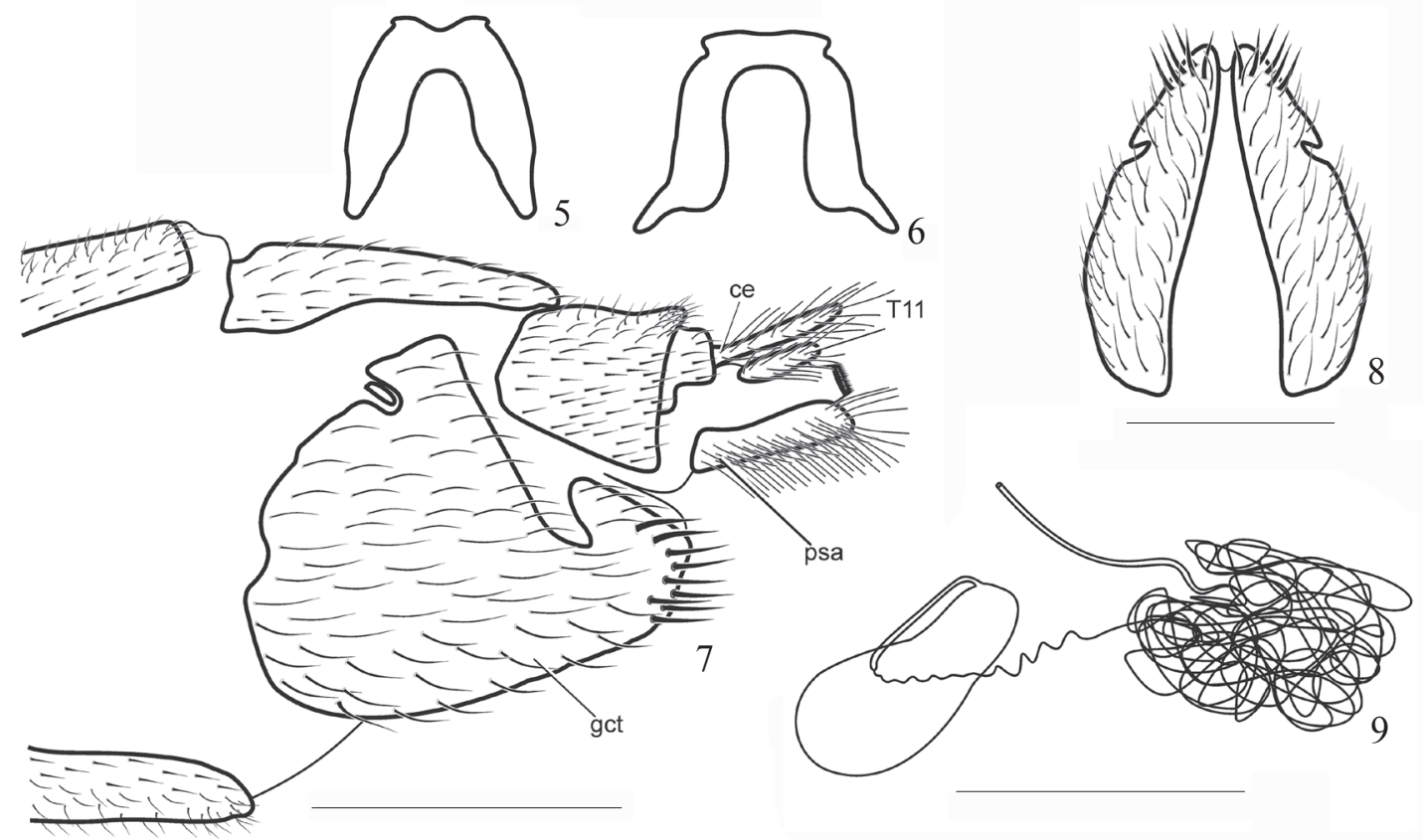

Figs. 5-9. 5, Bittacus diversinervis: epândrio, vista dorsal; 6, B. femoralis: epândrio, vista dorsal; 7-9: B. latreillei: 7, terminália, vista lateral, cerco (ce), gonocoxoesternitos (gct), placa sub-anal (psa), tergito $(\mathrm{T}) ; 8$, gonocoxoesternitos, vista ventral; 9, espermateca. Escalas $=1.0 \mathrm{~mm}$.

adultos só foram coletados nos meses mais chuvosos, de outubro a janeiro.

\section{CONCLUSÃO}

A bionomia das espécies brasileiras ainda é totalmente desconhecida, e o grande número de exemplares coletados, associado a sazonalidade bem estabelecida, é de grande importância para a coleta de outras espécies de mecópteros e para direcionar futuros estudos que almejam conhecer a bionomia das espécies locais. O esforço de coleta nas regiões mais áridas deve-se concentrar no período mais chuvoso.

Agradecimentos. À acadêmica de Ciências Biológicas do CESC/ UEMA Roseane O. Souza e às Biólogas Juciane C. Silva e Mery Jouse A. Holanda pela colaboração na coleta dos espécimes; ao entomólogo Fábio S. P. Godoi pelas dicas na elaboração do manuscrito; Agroindustrial Serra Grande - AGRO SERRA e à Cooperativa do Parque Estadual do Mirador COOPERMIRA pelo apoio logístico quando da realização das pesquisas de campo; Secretaria de Meio Ambiente do Maranhão - SEMA e IBAMA pelas autorizações de coletas (SISBIO - $\mathrm{n}^{\circ}$ 11419-1).

\section{REFERÊNCIAS}

Byers, G. W. 1965. New and uncommon Neotropical Mecoptera. Journal of the Kansas Entomological Society 38: 135-144.

Byers, G. W. 1972. A new genus of Mecoptera from Brazil. Journal of the Kansas Entomological Society 45: 341-346.

Byers, G. W. \& R. Thornhill. 1983. Biology of the Mecoptera. Annual Review of Entomology 28: 203-228.

Carpenter, F. M. 1931. The biology of the Mecoptera. Psyche 38: 41-55.

Collucci, E. \& D. S. Amorin. 2000. Three new species of Thyridates Navás, 1908 (Mecoptera, Bittacidae) from Brazil, with new combinations and

Received 11/03/2010; accepted 04/11/2010

Editor: Silvio Shigueo Nihei some comments about phylogenetic relationships within the genus. Contribuições Avulsas Sobre a História Natural do Brasil, Série Zoologia 21: 1-8.

Collucci, E. \& D. S. Amorin. 2001. Descrição de uma nova espécie de Thyridates Navás, 1908 de Governador Valadares, estado de Minas Gerais, Brasil e redescrição de T. femoralis (Klug, 1836) (Mecoptera, Bittacidae). Contribuições Avulsas Sobre a História Natural do Brasil, Série Zoologia 36: 1-6.

Dias, P. G. \&A. F. Kumagai (no prelo). Ocorrência, abundância e sazonalidade de Bittacus pintoi Lopes \& Mangabeira, 1942 (Bittacidae, Mecoptera) em duas áreas de conservação em Minas Gerais, Brasil. Lundiana.

GEPLAN/ UEMA. 2002. Atlas do Maranhão/Gerência de Estado de Planejamento e Desenvolvimento Econômico, Laboratório de Geoprocessamento-UEMA, São Luis, GEPLAN, 44 p.

Grimaldi, D. \& M. S. Engel. 2005. 12 Antliophora: Scorpionflies, Fleas, and True Flies, p. 468-480. In: Grimaldi, D. \& M. S. Engel (eds.). Evolution of the insects. Cambridge University Press, xiii $755 \mathrm{p}$.

Machado, R. J. P.; F. S. P. Godoi \& J. A. Rafael. 2009. Neotropical Mecoptera (Insecta): new generic synonimyes, new combinations, key to families and genera, and checklist of species. Zootaxa 2148: 27-38.

Morgante, J. S. 1967. Duas novas espécies de Bittacus Latreille, 1807 (Mecoptera, Bittacidae). Papéis Avulsos de Zoologia 20: 55-58.

Penny, N. D. 1977. Two new species of Bittacidae (Mecoptera) from the Amazon Basin. Acta Amazonica 7: 423-427.

Penny, N. D. \& J. R. Arias. 1982. Notes on Amazonian Bittacidae (Mecoptera) with the descriptions of two new species. Memórias do Instituto Oswaldo Cruz 77: 263-274.

Penny, N. D. \& J. R. Arias. 1983. A new species of Issikiella from the Amazon Basin (Mecoptera: Bittacidae). Acta Amazonica 13(3-4): 701-704.

Setty, L. R.1940. Biology and morphology of some north American Bittacidae (Order Mecoptera). The American Midland Naturalist 23: 257-353.

Souza-Lopes, H. \& O. Mangabeira F. 1942. Sobre algumas espécies brasileiras do gênero Bittacus Latr., 1807, com a descrição de três espécies novas (Panorpatae, Bittacidae). Revista Brasileira de Biologia 2: 331-341.

Tan, J. L. \& B. Z. Hua. 2009. Bicaubittacus, a new genus of the Oriental Bittacidae (Mecoptera) with descriptions of two new species. Zootaxa 2221: $27-40$.

Tan, J. L. \& B. Z. Hua. 2009. Terrobittacus, a new genus of the Chinese Bittacidae (Mecoptera) with descriptions of two new species. Journal of Natural History 43: 2937-2954. 